\title{
Selective Targeting of Bone and Cartilage with Multivalent Dendritic Polyanions
}

Sabine Reimann¹, Dominic Gröger ${ }^{1}$, Ngee Han Lim², Kai Licha ${ }^{3}$, Pia Welker ${ }^{3}$, Tobias Schneider ${ }^{4}$, Hideaki Nagase ${ }^{2}$, Peter Fratzl $\left.\right|^{5}$, Rainer Haag ${ }^{1}$

${ }^{1}$ Institute of Chemistry and Biochemistry, Freie Universität Berlin, Takustr. 3, 14195 Berlin, Germany ${ }^{2}$ Kennedy Institute of Rheumatology Division, Faculty of Medicine, Imperial College London, 1 Aspenlea Rd, London W6 8LH, UK ${ }^{3}$ Zentralinstitut für Laboratoriumsmedizin und Pathobiochemie, ChariteUniversitätsmedizin Berlin, CBF, Hindenburgdamm 30, 12203 Berlin, Germany ${ }^{4}$ Charité-Universitätsmedizin Berlin CBF, Klinik für Orthopädische Unfallund Wiederherstellungschirurgie Garystr. 5, D-14195, Berlin, Germany ${ }^{5}$ Max Planck Institute of Colloids and Interfaces Department of Biomaterials, 14424 Potsdam, Germany.

\section{Introduction}

In previous studies native and demineralized ovine bone was used to analyze the affinity of polyanions derived from dendritic polyglycerol (dPG)

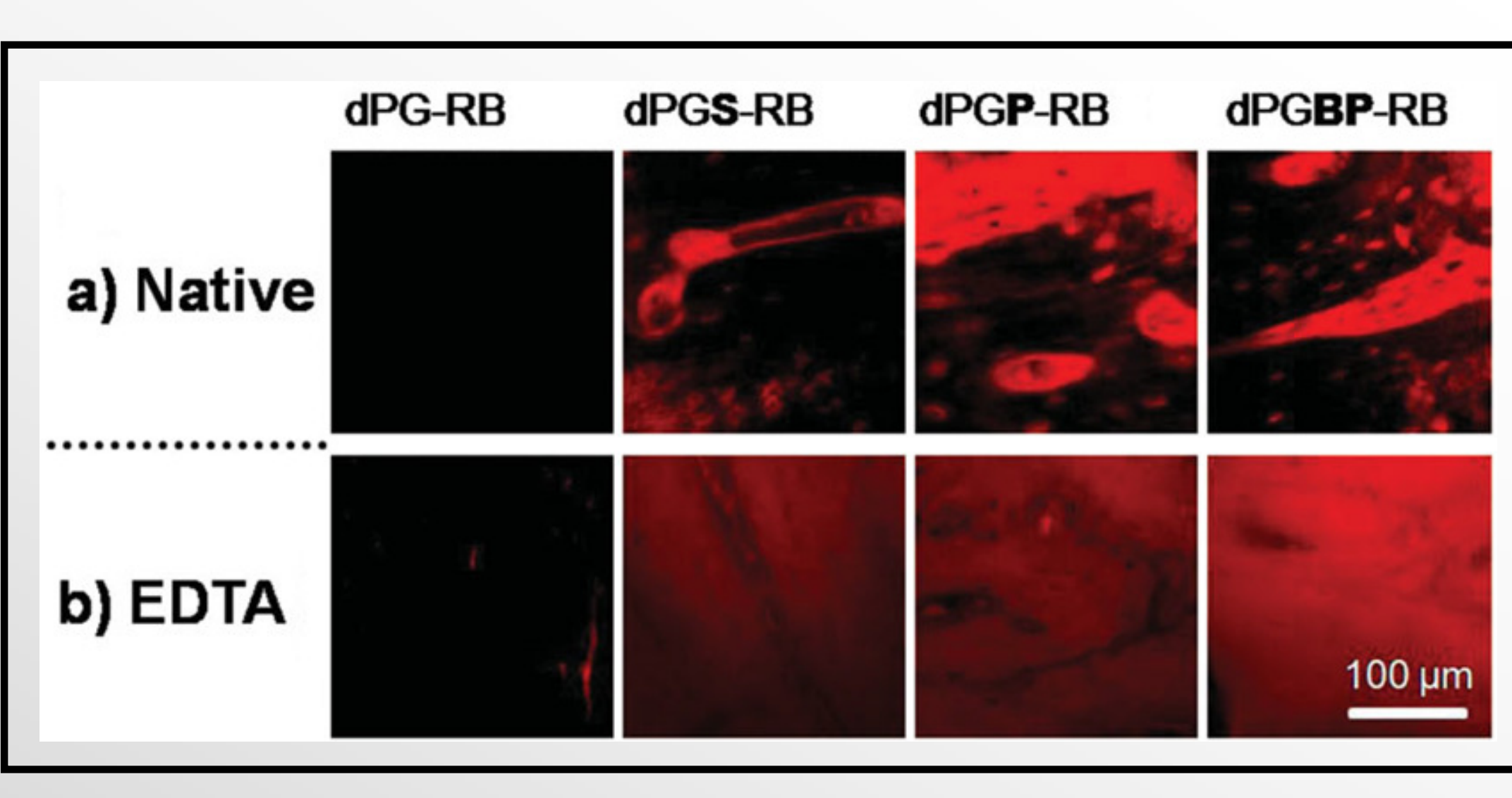

toward hydroxyapatite and collagen. [1] Whereas the neutral polymer did not show any interaction with bone, a selective binding to hydroxyapatite and collagen was observed depending on the anionic moiety of the polymer. Based on these results the binding affinity of polyanions toward cartilage was investigated to obtain selective targeting agents.

\section{Synthesis of Dye-Labeled Polyanions}

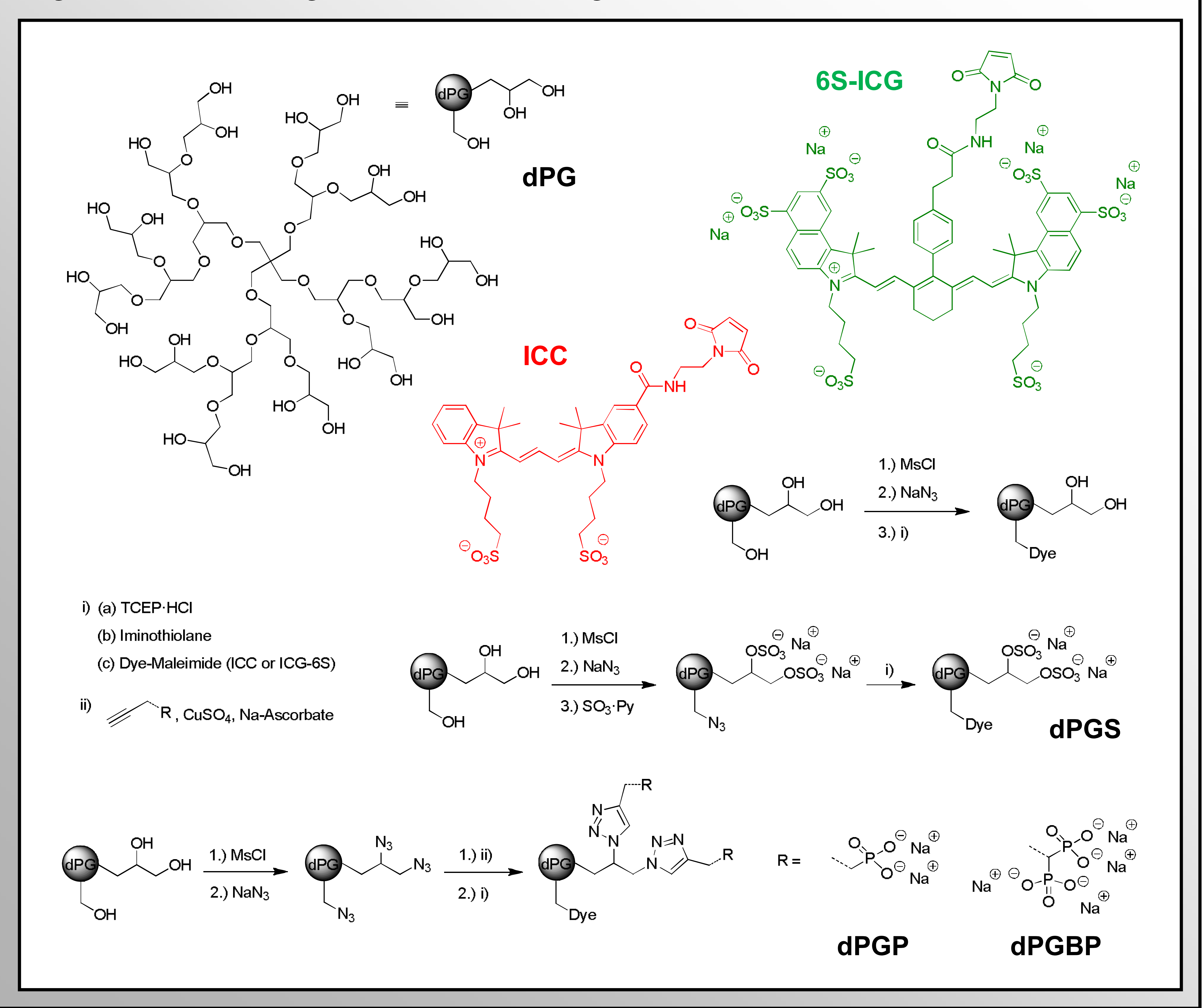

\section{Methods}

Rheumatoid arthritis was induced in female Lewis rats $(150 \pm 10 \mathrm{~g})$ by subcutaneous injection of collagen II after 1 and 8 days. NIR-fluorescence imaging in vivo was performed $24 \mathrm{~h}$ after one single i.v. injection of the 6S-ICG dye conjugates $(2 \mathrm{mg} / \mathrm{kg}$ ) via the tail vein. Animals included for later histology additionally received ICC conjugates $(1 \mathrm{mg} / \mathrm{kg})$. Histological recovery of the dye conjugates was analyzed by fluorescence microscopy. Histochemical staining was performed on cryo sections on cellotape of tibiotarsal articulations.

Sections of freshly explanted porcine cartilage were incubated with Interleukin$1 ß$ (IL-1ß) for $24 \mathrm{~h}$. Pictures were taken $24 \mathrm{~h}$ after incubation with 6 S-ICG labeled conjugates $\left(10^{-6} \mathrm{~mol} \mathrm{~L}^{-1}\right)$ by fluorescence microscopy. For glycosaminoglycan (GAG) release studies similar sized discs of the cartilage explants were incubated with IL-1ß alone or IL-1ß and $d P G$ or $d P G$ anions, respectively for $24 \mathrm{~h}$. Afterwards proteinase $\mathrm{K}$ was added and the total concentration of GAGs of the supernatant after centrifugation was determined by the dimethyl methylene blue method (DMMB). Untreated cartilage served as control.

\section{References}

[1] D. Gröger, M. Kerschnitzki, M. Weinhart, S. Reimann, T. Schneider, B. Kohl, W. Wagermaier G. Schulze-Tanzil, P. Fratzl, R. Haag, Advanced Healthcare Materials 2013, 3, 375-385

[2] K. Licha, P. Welker, M. Weinhart, N. Wegner, S. Kern, S. Reichert, I. Gemeinhardt, C. Weissbach, B. Ebert, R. Haag, M. Schirner, Bioconjugate Chem. 2011, 22, 2453-2460.

\section{Binding Affinity to Joints in Vivo}

Dendritic polyglycerol sulfate (dPGS) known as antiinflammatory agent accumulates in inflamed joints in rheumatoid arthritis (CIA). [2] Using the same animal model, bisphosphonate

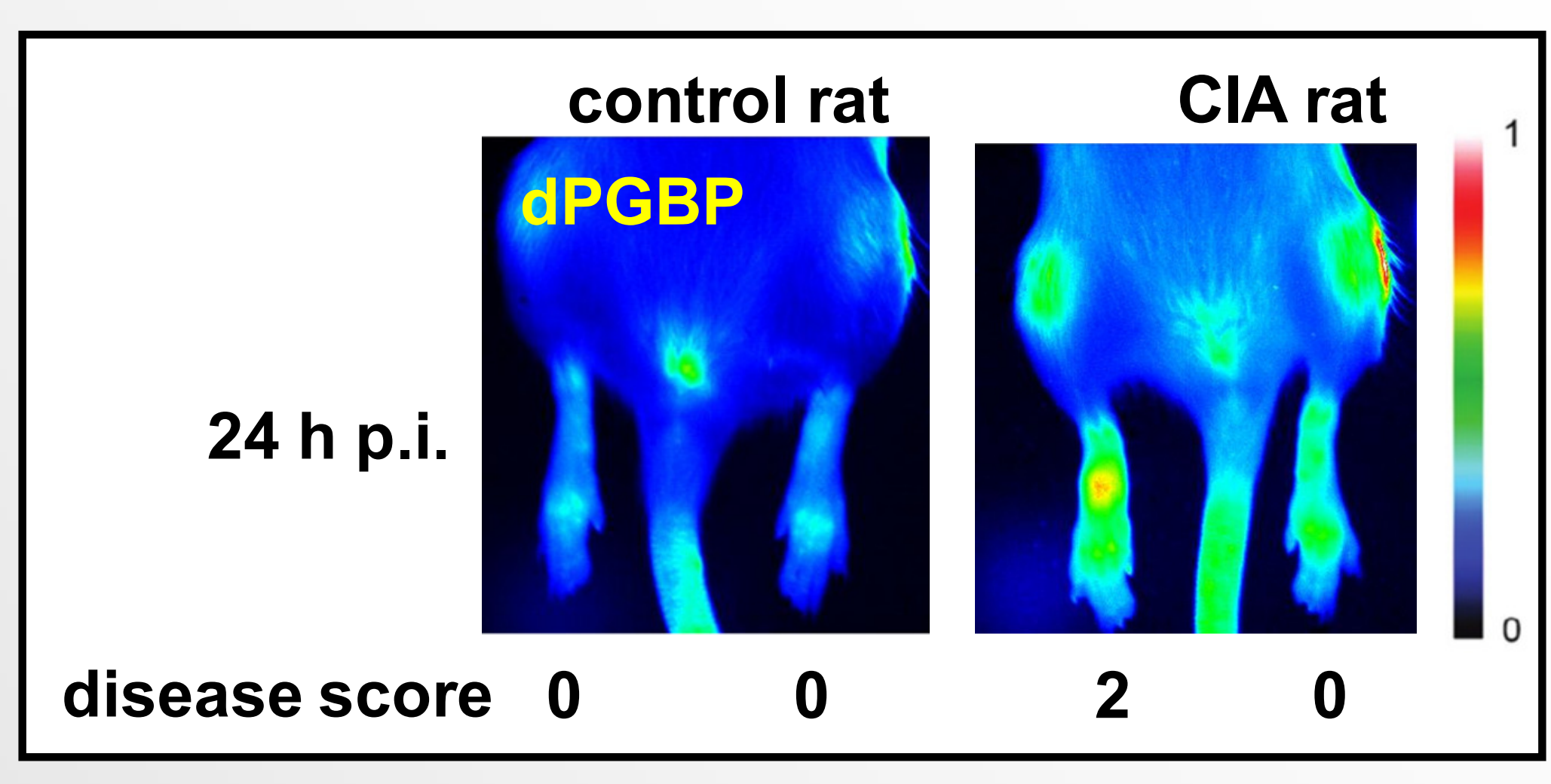
modified dPG (dPGBP) also shows an affinity to inflamed tissue.

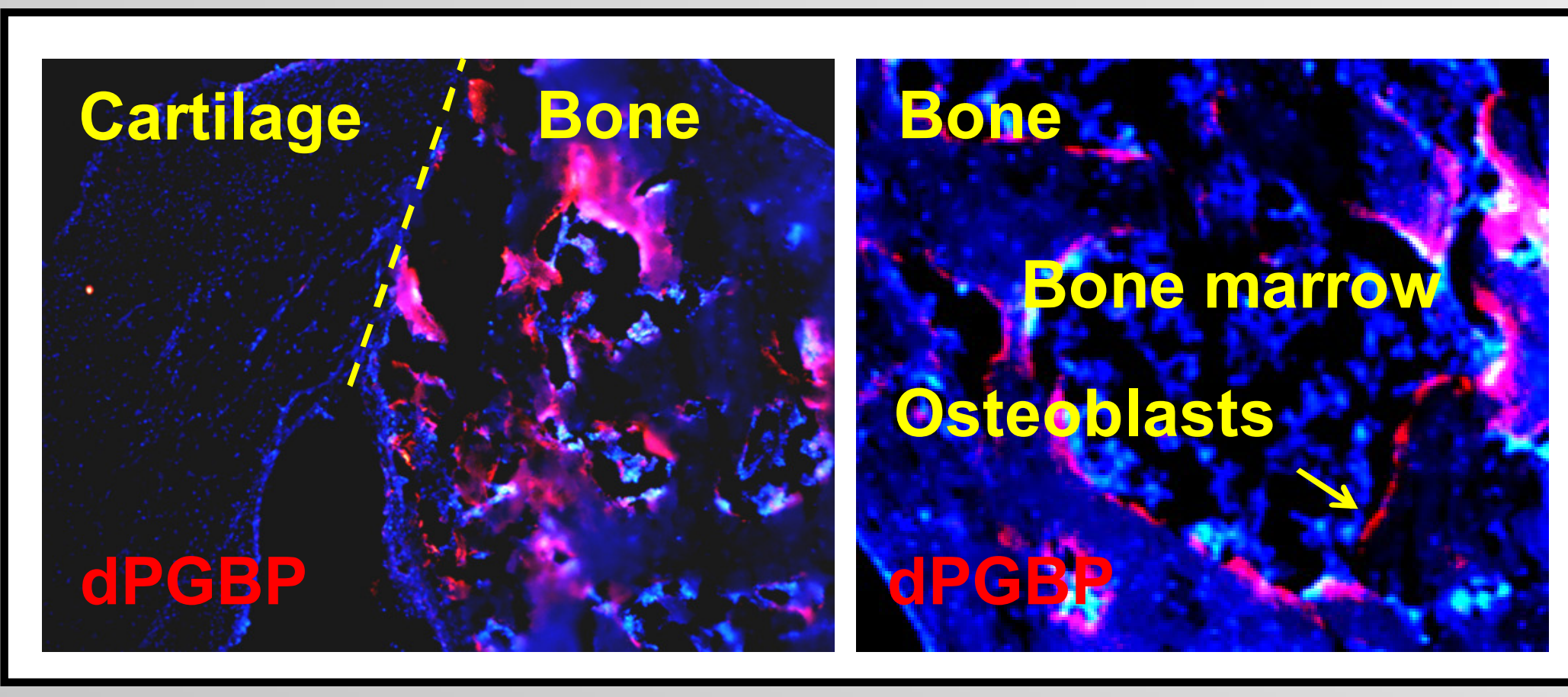

Histological recovery of ICC labeled dPG-BP in healthy rat tissue showed enrichment only in bone, accumulating in osteoblasts.

In CIA rat tissue both dPGS and dPGBP were found to bind to inflamed cartilage.

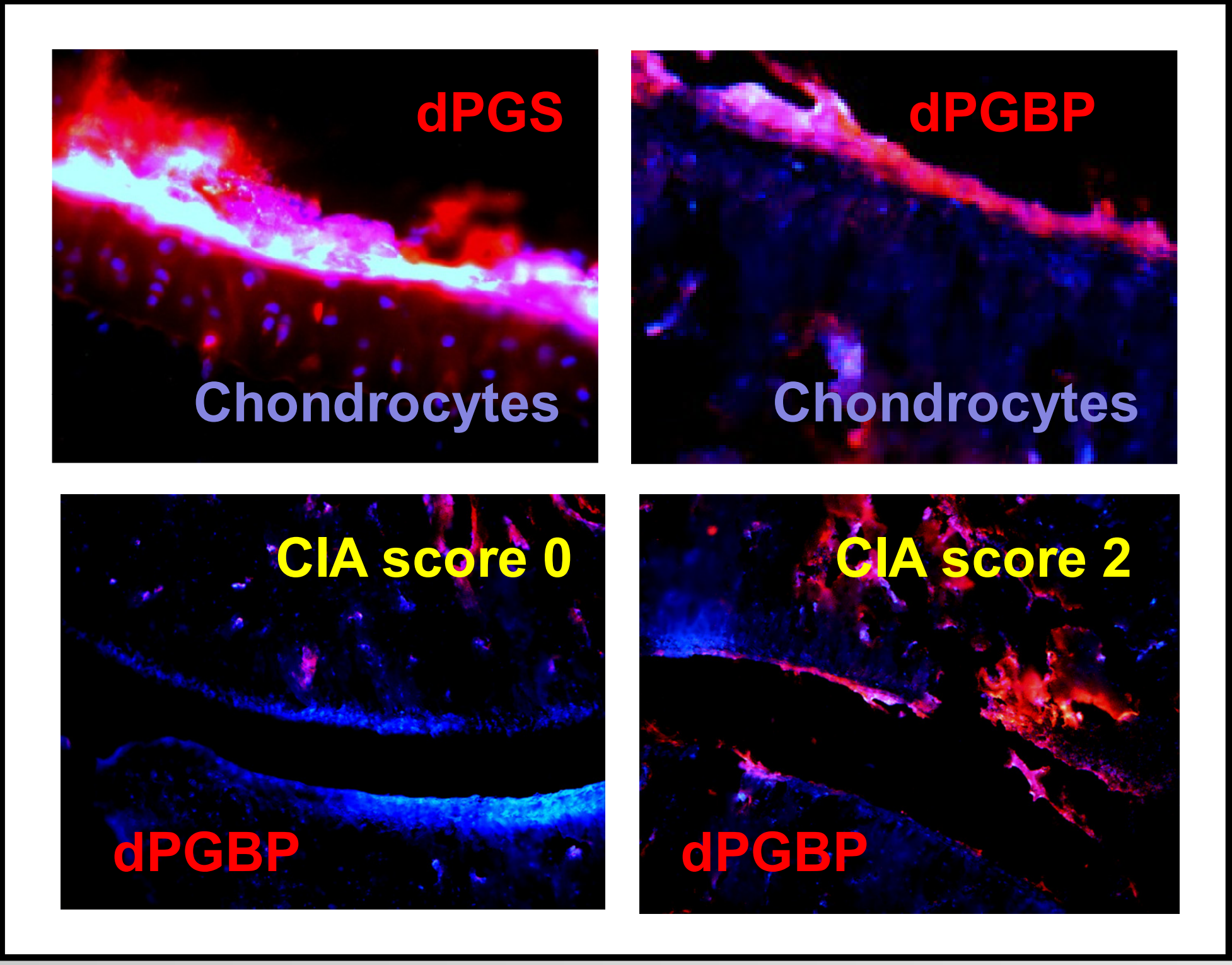

Interaction with IL-1 Treated Explanted Cartilage

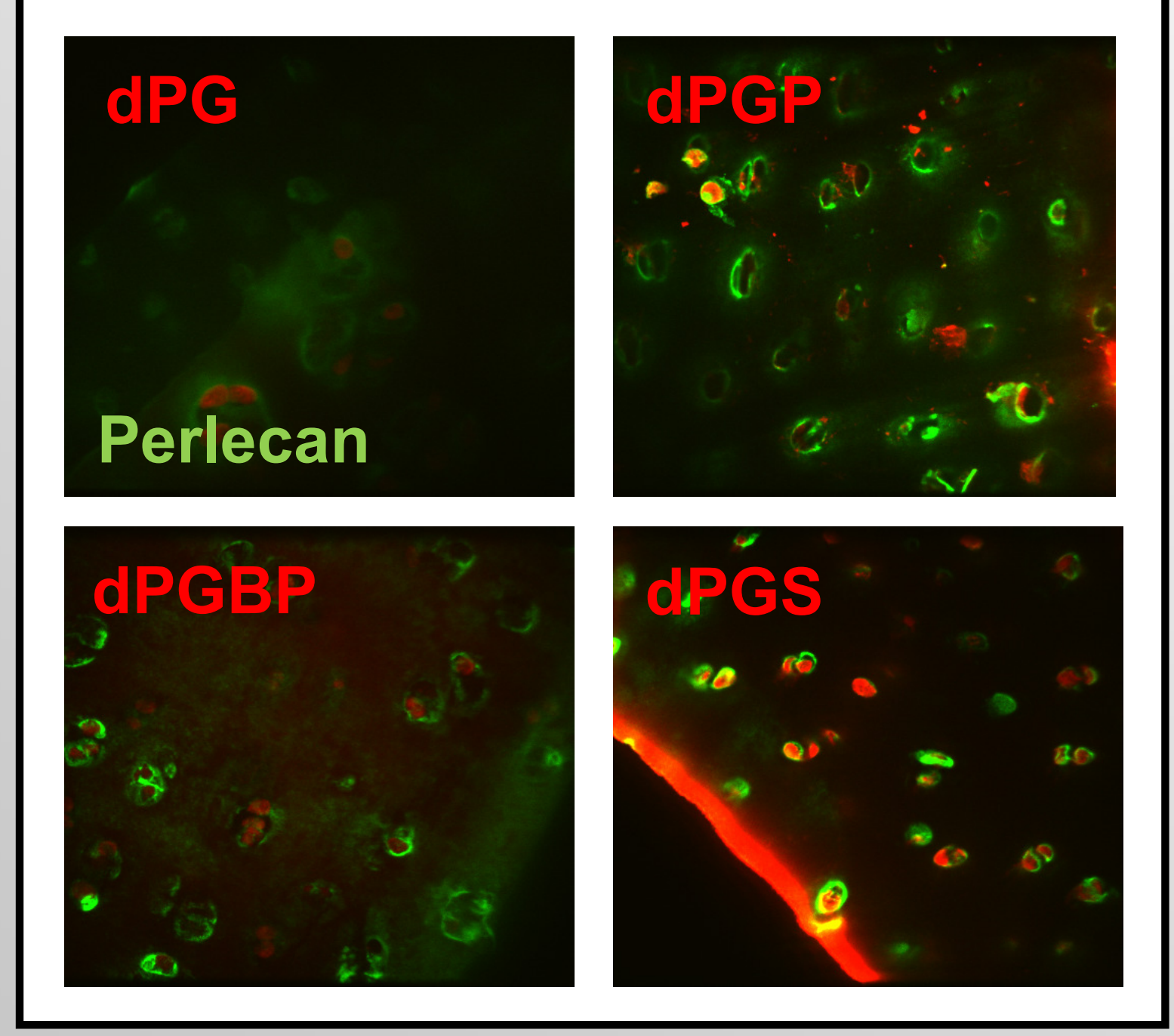

Investigating the binding affinity to Interleukin 1 treated porcine cartilage explants, dPGS was found to efficiently penetrate the cartilage tissue and accumulate in chondrocytes.

When investigating the inhibition of glycosaminoglycan (GAG) release dPGS and dPGBP were found to efficiently reduce the GAG loss.

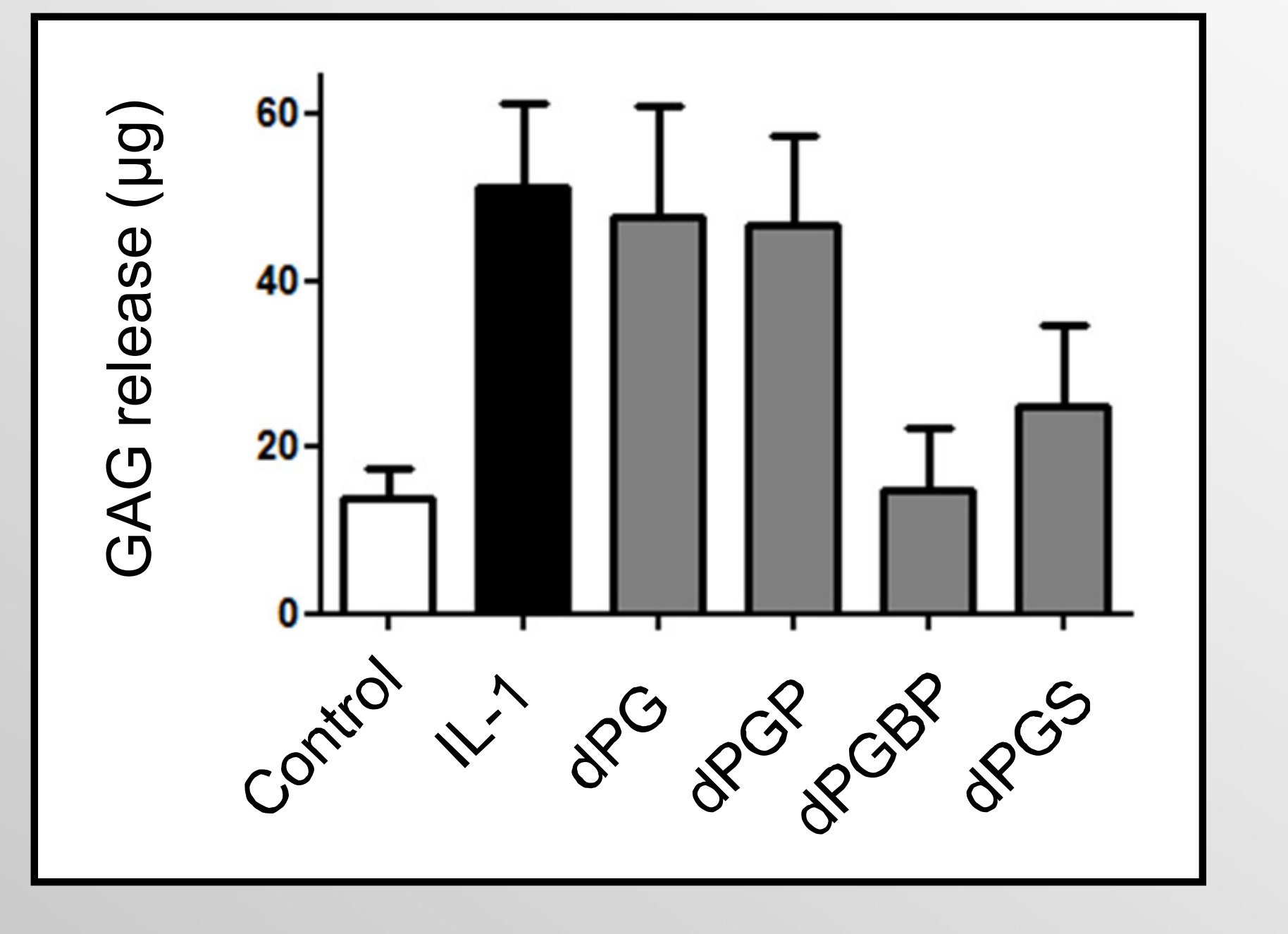

\section{Conclusion}

Dendritic polyglycerol based anions were found to interact with bone and cartilage depending on the nature of the anionic group and the disease. dPG anion derivatives are therefore considered as promising candidates for diagnostic and therapeutic applications to target healthy and malfunctioning bone and cartilage.

\section{Acknowledgements}

We thank all co-authors for scientific contributions and the DFG, SFB765, and IMPRS on Multiscale Biosystems for financial support. 